\title{
Selection Diversity in General Scattering Environments
}

\author{
Jaunty T.Y. Ho, Rodney A. Kennedy and Thushara D. Abhayapala \\ Department of Telecommunications Engineering \\ RSISE, The Australian National University \\ Canberra ACT 0200, Australia \\ \{jaunty.ho, rodney.kennedy, Thushara.Abhayapala\}@anu.edu.au
}

\begin{abstract}
We derive an infinite series expression of the average signal-to-noise ratio (SNR) gain for a dual selection combining system under Nakagami- $m$ fading using a series representation of the bivariate cumulative probability distribution function. The average SNR gain expression is shown to relate to the Nakagami shape factor $m$, the power correlation and the power allocation ratio.
\end{abstract}

\section{INTRODUCTION}

T HE use of multi-element antennas can increase the performance of wireless systems by utilising spatial diversity. By assuming independence between the received signals from various antennas, the performance of common multi-element antennas systems, such as maximal ratio combining, equal gain combining and selection combining, have been reported $[1,2]$. Recently, the performance of a generalized diversity selection combining system has also been investigated [3]. However, the independence assumption between received signals at different antennas is not generally valid. An example is the limited separation of antennas in small wireless mobile units. The spatial diversity cannot be fully utilized due to spatial correlation between received signals. Therefore, the performance of the multi-element antenna systems cannot be realistically investigated with the independence assumption.

The spatial correlation between received signals from different antennas should be considered. Under the assumption of Rayleigh fading, the expression for the cumulative probability distribution (also called outage probability) of the maximum ratio combining systems with multi-element antennas has been derived in [1]. The corresponding expressions of the equal gain and the selection combining systems have been limited to dual antennas systems only, due to the difficulty of defining the joint probability distribution function for systems with more than two antennas $[1,2]$.

Dual selection combining systems generally receive greater attention in the literature. It is one of the least complicated diversity systems, not requiring co-phasing on each received signal. The selection combining scheme can be used in conjunction with differentially coherent and noncoherent modulation techniques. The strategy in dual selection combining is to select and use only the antenna that has the higher SNR, at any time instant, and discard the signal from the other antenna. Whilst this represents a loss of information, this strategy has many benefits and the performance loss relative to more optimal strategies need not be great. That is, in a multipath fading environment this strategy largely protects the receiver, in a probabilistic sense, from deep fades. Selection diversity systems also have a simplicity comparable to single antenna systems, making them attractive from an implementation viewpoint.

As previously mentioned, the Rayleigh bivariate cumulative probability distribution function for calculating the outage probability of the dual selection combining system has been derived [1]. However, the expression is in terms of the Marcum $Q$-function, which is not a mathematically convenient function and is not available in standard versions of mathematical software packages such as Maple V, MATLAB and Mathematica. Therefore, two main alternative representations of the cumulative probability distribution functions have been introduced: one is in terms of infinite series representation [4] and the other is in terms of a single integral representation [5]. Those alternative representations can also be extended from Rayleigh fading to Nakagami- $m$ fading. Using the alternative expressions, the performance metrics such as outage probability, average error probability and bit-error rate (BER), are reported for the dual selection combining systems [6]. Moreover, an expression for the average signal-to-noise ratio (SNR) of dual selection combining over correlated Nakagami-m fading systems is introduced in [7]. Apart from a few special cases, the expression is quite complicated, making it hard to see the mathematical structures and patterns [7].

Independent from previous work in [7], in this paper, we derive an alternative close form SNR expression of dual selection combining over correlated Nakagami- $m$ fading systems. The derivation is based on the infinite series representation of the cumulative probability distribution function [4]. Our expression gives a more compact series expression. It also introduces another application of the alternative infinite series representation from [4].

The paper is organized as follows. In Section II, we derive a series expression of the average SNR gain of the correlated dual selection combining system under Nakagami fading. In fact, the expression describes the average SNR gain of any correlated dual selection combining system under Nakagami fading; that is, two received signals can be correlated in time, frequency or space. Moreover, Rayleigh fading, also considered in the paper, is a special case of Nakagami fading. In Section III, we show that only a limited number of terms are required in the series expression of the average SNR gain for accurate numerical analysis. In Section IV, we quantitatively relate the average SNR gain to the different Nakagami shape 
factor $m$, the power correlation $\rho$ and the power allocation ratio $r$. We conclude the paper in Section $\mathrm{V}$ and the derivation of the average SNR gain expression is given in the Appendix.

\section{NAKAGAMI-M FADINO}

Define the received signal at antenna $i=\{1,2\}$ as $s_{i}$, and its corresponding signal-to-noise ratio (SNR) as $y_{i}^{2}=$ $\left|s_{i}\right|^{2} /\left(2 N_{i}\right)$, where $N_{i}$ is the Additive White Gaussian Noise (AWGN) at antenna $i, y_{i}>0$. For the dual selection system, only the signal with the stronger SNR is selected, at any time instant; and the weaker signal is discarded. Therefore, the selected signal power is given by $z \triangleq \max \left\{y_{1}^{2}, y_{2}^{2}\right\}$.

The joint Nakagami- $m$ probability distribution function (pdf) of two randorn vectors $y_{1}$ and $y_{2}$ is [8]

$$
\begin{gathered}
f_{y_{1}, y_{2}}\left(y_{1}, \Omega_{1} ; y_{2}, \Omega_{2} \mid m, \rho\right)=I_{m-1}\left\{\frac{2 \sqrt{\rho} y_{1} y_{2}}{\sqrt{\Omega_{1} \Omega_{2}}(1-\rho)}\right\} \\
\times \frac{4\left(y_{1} y_{2}\right)^{m} e^{-\left(\Omega_{2} r_{1}^{2}+\Omega_{1} r_{2}^{2} / \Omega_{1} \Omega_{2}(1-\rho)\right)}}{\Gamma(m) \Omega_{1} \Omega_{2}(1-\rho)\left(\sqrt{\Omega_{1} \Omega_{2} \rho}\right)^{m-1}}
\end{gathered}
$$

where $I_{m}(\cdot)$ is the modified bessel functions of the first kind, $\Gamma(m)=\int_{0}^{\infty} t^{m-1} e^{-t} d t, \operatorname{Re}(m)>0$ is the Euler's gamma function ${ }^{1}, \Omega_{i}=E\left\{y_{i}^{2}\right\} / m, m$ is the Nakagami shape factor (any positive number not less than $1 / 2$ ) and

$$
\rho=\frac{\operatorname{cov}\left(y_{1}^{2}, y_{2}^{2}\right)}{\sqrt{\operatorname{var}\left(y_{1}^{2}\right) \operatorname{var}\left(y_{2}^{2}\right)}}, \quad \rho \neq 0,1
$$

is the power correlation of the received signal. Notice that for the special case of $m=1$, (1) becomes the bivariate probability density function (pdf) of two correlated Rayleigh random variables, derived in [9]. Furthermore, the outage probability (also known as the cumulative distribution function, cdf) of the SNR $z$ below some value $\gamma$ is defined as

$$
\begin{aligned}
\operatorname{Pr}(z<\gamma) & =\operatorname{Pr}\left(y_{1}<\sqrt{\gamma}, y_{2}<\sqrt{\gamma}\right) \\
& \triangleq F_{y_{1}, y_{2}}\left(\sqrt{\gamma}, \Omega_{1} ; \sqrt{\gamma}, \Omega_{2} \mid m, \rho\right) .
\end{aligned}
$$

where $F_{y_{1}, y_{2}}(\cdot)$ is the cdf of $z$. With the use of infinite series representations derived in [4], the outage probability of $z$ becomes

$$
\begin{array}{r}
\operatorname{Pr}(z<\gamma)=\frac{(1-\rho)^{m}}{\Gamma(m)} \sum_{k=0}^{\infty} \rho^{k}\{ \\
\left.\times \frac{G\left(m+k, \frac{\gamma}{\Omega_{1}(1-\rho)}\right) G\left(m+k, \frac{\gamma}{\Omega_{2}(1-\rho)}\right)}{k ! \Gamma(m+k)}\right\}
\end{array}
$$

where

$$
G(n, x)=\int_{0}^{x} t^{n-1} e^{-t} d t, \quad \operatorname{Re}(n)>0
$$

${ }^{1}$ If $m$ is an positive integer, then $\Gamma(m)=(m-1)$ !. is the incomplete gamma function [10]. This expression contrasts with classical results expressed in terms of the Marcum Q-function $[1,9]$. With this expression, we derive an alternative expression for the average SNR of the correlated dual selection combining system under Nakagami- $m$ fading.

The average (expected value) SNR of the system is defined as

$$
E\{z\} \triangleq \int_{0}^{\infty} \gamma f_{z}\{\gamma\} d \gamma
$$

where

$$
f_{z}(\gamma)=\frac{d}{d \gamma} \operatorname{Pr}(z<\gamma)
$$

is the pdf of $z$. After some simplification, we find

$$
\begin{aligned}
f_{z}(\gamma)= & \sum_{k=0}^{\infty}\left\{\xi\left(\gamma, \Omega_{1}, \rho, m, k\right) G\left(m+k, \frac{\gamma}{(1-\rho) \Omega_{2}}\right)\right. \\
& \left.+\xi\left(\gamma, \Omega_{2}, \rho, m, k\right) G\left(m+k, \frac{\gamma}{(1-\rho) \Omega_{1}}\right)\right\}
\end{aligned}
$$

where

$$
\xi\left(\gamma, \Omega_{i}, \rho, m, k\right)=\frac{\rho^{k} \gamma^{m+k-1} e^{-\frac{\gamma}{(1-\rho) \Omega_{i}}}}{(1-\rho)^{k} k ! \Omega_{i}^{m+k} \Gamma(m+k) \Gamma(m)} .
$$

Substituting (8) into (6), and using the results in the Appendix, we show that the new average SNR expression is

$$
\begin{aligned}
& E\{z\}=\frac{\Omega(1-\rho)^{m+1} r^{m+1}}{\Gamma(m+1)(1+r)^{2 m+1}} \sum_{k=0}^{\infty}\{ \\
& \left.\frac{\rho^{k} r^{k}}{k !(1+r)^{2 k}} \sum_{n=0}^{\infty} \frac{\Gamma(2 m+2 k+n+1)}{\Gamma(m+k+n+1)} \frac{\left(1+r^{n}\right)}{(1+r)^{n}}\right\}
\end{aligned}
$$

where $\Omega$ is related to $\Omega_{1}$ and $\Omega_{2}$ through

$$
\begin{aligned}
& \Omega_{1}=\frac{E\left\{y_{1}^{2}\right\}}{m}=\frac{\Omega}{m} \\
& \Omega_{2}=\frac{E\left\{y_{2}^{2}\right\}}{m}=\frac{r \Omega}{m}
\end{aligned}
$$

by introducing the power allocation ratio $r=\Omega_{2} / \Omega_{1}$. Expression (10) is an infinite series which allows us to determine the average SNR of $z$. The average SNR depends on power correlation $\rho$, the Nakagami shape factor $m$, the power allocation ratio $r$ and $\Omega$. Now we can determine the average SNR gain of a dual selection system under Nakagami-m channel fading. Define the average SNR gain in $\mathrm{dB}$ as

$$
\operatorname{Gain}_{\mathrm{dB}}(\rho, m, r) \triangleq 10 \log _{10}\left\{\frac{E\{z\}}{\Omega}\right\} .
$$


By substituting (10) into (13), we obtain

$$
\begin{aligned}
& \operatorname{Gain}_{\mathrm{dB}}(\rho, m, r)=10 \log _{10}\left\{\frac{(1-\rho)^{m+1} r^{m+1}}{\Gamma(m+1)(1+r)^{2 m+1}}\right. \\
& \left.\times \sum_{k=0}^{\infty} \frac{\rho^{k} r^{k}}{k !(1+r)^{2 k}} \sum_{n=0}^{\infty}\left(\frac{\Gamma(2 m+2 k+n+1)}{\Gamma(m+k+n+1)} \frac{\left(1+r^{n}\right)}{(1+r)^{n}}\right)\right\} .
\end{aligned}
$$

The average SNR gain depends on the power correlation $\rho$, the Nakagami shape factor $m$ and the power allocation ratio $r=$ $\Omega_{2} / \Omega_{1}$. With such an infinite series expression of average SNR gain, we can now relate the average SNR gain with different $\rho$, $m$ and $r$ analytically in Section IV.

\section{TRUNCATEd SERIES EXPRESSION}

Equation (14) gives a series expression, with two infinite sums, for the average SNR gain. However, due to the structure of (14), it is apparent that the sum is monotonically decreasing with $n$ or $k$. We would like to find the required number terms of $n$ and $k$ for the truncated series expression to be sufficient close to the full series with acceptable error. Since the new terms reduce its significance monotonically, we ignore the new term when its value (in $E\{z\} / \Omega$ ) is less than $10^{-9}$. Table I shows the number of terms $n$ and $k$ required with a different power correlation $\rho$, Nakagami shape fading $m$ and power allocation ratio $r$. By doing this, we found that the first 1000 terms of $n$ and $k$ are more than enough to accurately calculate the average SNR gain.

\section{RESULTS}

The series expression (14) can quantitatively relate the average SNR gain to any power correlation $\rho$, Nakagami shape factor $m$ and power allocation ratio $r$. Figs. 1 and 2 show the average SNR gain of the dual selection system with a different Nakagami shape factor $m=\{0.5,0.7,1,2.2\}$ and with a different power allocation ratio $r=\Omega_{2} / \Omega_{1}=\{1,2\}$. For the numerical calculations, we used only the first 1000 terms of $\mathrm{k}$ and $\mathrm{n}$ to generate Figs. 1 and 2. The results are consistent with those of [7]. It is also noticed that two trivial results $[1,3]$, when two received signals are fully correlated $(\rho=1)$ or uncorrelated $(\rho=0)$, are covered in our series expression. The figures show that if $m$ increases, the average SNR gain decreases. The received signal suffers less fading and therefore has less signal variation. Thus it achieves less average SNR gain. Moreover, once the branch average SNR is imbalanced, say $r=2$, the average SNR gain will also decrease; since there is a greater chance that the antenna with higher local SNR average will be selected, this reduces the diversity of the system. It is shown in Figs. 1 and 2 that for Rayleigh fading $(m=1)$, when one local average SNR is the twice that of the other, the available average SNR gain ( $\rho=0)$ drops by $1.28 \mathrm{~dB}$ (from $1.761 \mathrm{~dB}$ to $0.6695 \mathrm{~dB}$ ), compared to the balance average SNR. Similarly, we can determine the average SNR gain difference for all different $m, \rho$ and $r$.
TABLE I

THE NUMBER OF TERMS $n$ AND $k$ REQUIRED SUCH THAT ALL EXCLUDED TERMS ARE NOT GREATER THAN $10^{-9} \cdot(m, r)$ REPRESENTS THE NAKAGAMI SHAPE FADING $m$ AND POWER ALLOCATION RATIO $r$.

\begin{tabular}{|c|c|c|c||c|c|c|c|}
\hline$(1,1)$ & $\rho$ & $n$ & $k$ & $(1,2)$ & $\rho$ & $n$ & $k$ \\
\hline & 0.1 & 34 & 11 & & 0.1 & 56 & 10 \\
\hline & 0.3 & 36 & 19 & & 0.3 & 62 & 18 \\
\hline & 0.5 & 39 & 31 & & 0.5 & 73 & 29 \\
\hline & 0.7 & 46 & 57 & & 0.7 & 98 & 53 \\
\hline & 0.9 & 66 & 173 & & 0.9 & 206 & 161 \\
\hline$(2,1)$ & $\rho$ & $n$ & $k$ & $(2,2)$ & $\rho$ & $n$ & $k$ \\
\hline & 0.1 & 37 & 11 & & 0.1 & 61 & 11 \\
\hline & 0.3 & 39 & 21 & & 0.3 & 68 & 19 \\
\hline & 0.5 & 42 & 34 & & 0.5 & 81 & 32 \\
\hline & 0.7 & 49 & 63 & & 0.7 & 109 & 60 \\
\hline & 0.9 & 71 & 196 & & 0.9 & 223 & 183 \\
\hline$(10,1)$ & $\rho$ & $n$ & $k$ & $(10,2)$ & $\rho$ & $n$ & $k$ \\
\hline & 0.1 & 49 & 15 & & 0.1 & 91 & 15 \\
\hline & 0.3 & 52 & 30 & & 0.3 & 102 & 29 \\
\hline & 0.5 & 57 & 53 & & 0.5 & 124 & 51 \\
\hline & 0.7 & 68 & 103 & & 0.7 & 173 & 98 \\
\hline & 0.9 & 101 & 334 & & 0.9 & 394 & 318 \\
\hline
\end{tabular}

\section{CONClusion}

An alternative series expression of the average SNR gain of the dual selection combining system, under Nakagami fading, was derived based on the infinite series representations of the bivariate Nakagami-m distribution [4] . The average SNR gain is a function of power correlation $\rho$, Nakagami shape factor $m$ and the power allocation ratio $r$. Generally, the alternative expression itself is more compact than the equivalent expression in [7].

\section{REFERENCES}

[1] W. C. Jakes, Microwave Mobile Communications, New York: Wiley, 1974

[2] M. Schwartz, W. Bennett, and S. Stein, Communication Systems and Techniques, New York: McGraw-Hill Book Company, 1965.

[3] N. Kong and L. B. Milstein, "Average SNR of a generalized diversity selection combining scheme," IEEE Commun. Lett., vol. 3, pp. 57-59, March 1999.

[4] C. C. Tan and N. C. Beaulieu, "Infinite series representations of the bivariate Rayleigh and Nakagami-m distributions," IEEE Trans. on Communications, vol. 45, pp. 1159-1161, Oct. 1997.

[5] M. K. Simon and M.-S. Alouini, "A simple single integral representation of the bivariate Rayleigh distribution," IEEE Commun. Lett, vol. 2, no. 5, pp. 128-130, May 1998.

[6] M. K. Simon and M-S. Alouini, "A unified performance analysis of digital communication with dual selective combining diversity over correlated Rayleigh and Nakagami-m fading channels," IEEE Trans. on Communications, vol. 47, no. 1, pp. 33-43, Jan. 1999.

[7] Y.-C. Ko, M.-S. Alouini, and M. K. Simon, "Average SNR of dual selection combining over correlated Nakagami-m fading channels," IEEE Commun. Lett., vol. 4, no. 1, pp. 12-14, Jan. 2000.

[8] M. Nakagami, "The m-distribution- a general formula of intensity distribution of rapid fading," Statistical Methods in Radio Wave Propagation, 1960. 


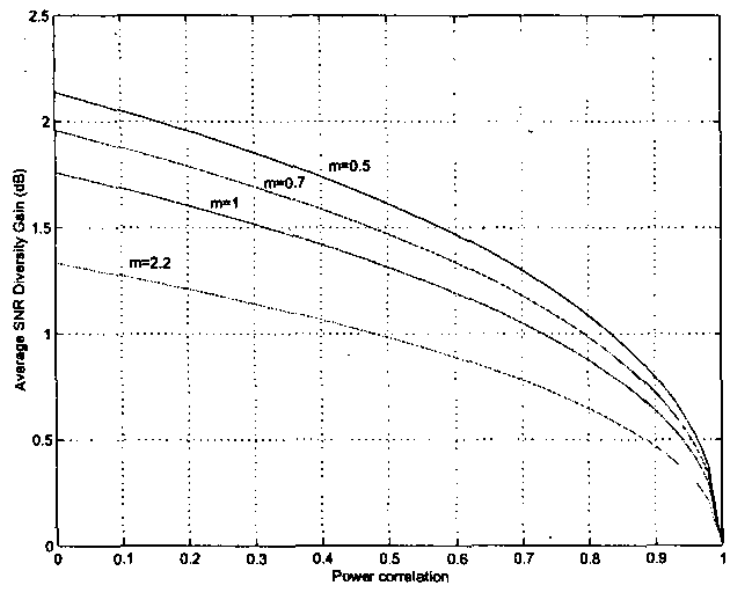

Fig. 1. Dual Selection Diversity Gain (dB) vs Power Correlation. The power allocation ratio $r=\Omega_{2} / \Omega_{1}=1$ and the Nakagami shape factor $m=\{0.5,0.7,1,2.2\}$.

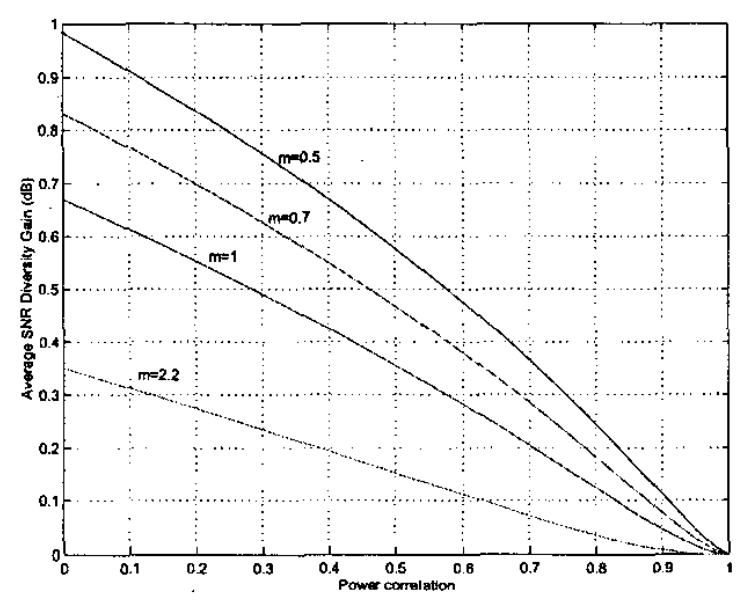

Fig. 2. Dual Selection Diversity Gain (dB) vs Power Correlation. The power allocation ratio $r=\Omega_{2} / \Omega_{1}=2$ or 0.5 and the Nakagami shape factor $m=\{0.5,0.7,1,2.2\}$.

[9] W. B. Davenport and W. L. Root, Introduction to Random Signals and Noise, New York: McGraw Hill, 1958.

[10] I. S. Gradshteyn and I. M. Ryzhik, Table of Intergrals, Series, and Noise, United Kingdom: Acadernic Press Limited, Sth edition, 1994.

\section{APPENDIX}

Substitute (8) into (6), the average SNR becomes

$$
\begin{aligned}
E\{z\}= & \int_{0}^{\infty} \gamma \sum_{k=0}^{\infty}\left\{\xi\left(\gamma, \Omega_{1}, \rho, m, k\right)\right. \\
& \left.\times G\left(m+k, \frac{\gamma}{(1-\rho) \Omega_{2}}\right)\right\} d \gamma \\
& +\int_{0}^{\infty} \gamma \sum_{k=0}^{\infty}\left\{\xi\left(\gamma, \Omega_{2}, \rho, m, k\right)\right. \\
& \left.\times G\left(m+k, \frac{\gamma}{(1-\rho) \Omega_{1}}\right)\right\} d \gamma \\
= & A\left(\gamma, \Omega_{1}, \rho, m, k, \Omega_{2}\right)+A\left(\gamma, \Omega_{2}, \rho, m, k, \Omega_{1}\right)
\end{aligned}
$$

where

$$
\begin{aligned}
& A\left(\gamma, \Omega_{i}, \rho, m, k, \Omega_{j}\right) \\
& =\int_{0}^{\infty} \gamma \sum_{k=0}^{\infty}\left\{\xi\left(\gamma, \Omega_{i}, \rho, m, k\right) G\left(m+k, \frac{\gamma}{(1-\rho) \Omega_{j}}\right)\right\} d \gamma \\
& =\sum_{k=0}^{\infty}\left\{\frac{\rho^{k}}{(1-\rho)^{k} k ! \Omega_{i}^{m+k} \Gamma(m+k) \Gamma(m)}\right. \\
& \quad \times \int_{0}^{\infty} \gamma^{m+k} e^{\left.-\frac{\gamma}{(1-\rho) \Omega_{i}} G\left(m+k, \frac{\gamma}{(1-\rho) \Omega_{j}}\right) d \gamma\right\}}
\end{aligned}
$$

We can further simplify (16) by using $[10,(6.455)]$,

$$
\begin{array}{r}
\int_{0}^{\infty} x^{\mu-1} e^{-\beta x} G(v, \alpha x) d x=\frac{\alpha^{v} \Gamma(\mu+v)}{v(\alpha+\beta)^{\mu+v}} \\
\times F\left(1, \mu+v ; v+1 ; \frac{\alpha}{\alpha+\beta}\right)
\end{array}
$$

where $\operatorname{Re}(\alpha+\beta)>0, \operatorname{Re}(\beta)>0, \operatorname{Re}(\mu+v)>0$ and $F(\cdot, ; \cdot ; \cdot)$ is the Gauss hypergeometric function. Therefore, using the result of (17), (16) becomes

$$
\begin{aligned}
A\left(\gamma, \Omega_{i}, \rho, m, k, \Omega_{j}\right)=\frac{(1-\rho)^{m+1} \Omega_{i}^{m+1} \Omega_{j}^{m+1}}{\left(\Omega_{i}+\Omega_{j}\right)^{2 m+1} \Gamma(m)} \\
\quad \times \sum_{k=0}^{\infty}\left\{\frac{\Omega_{i}^{k} \Omega_{j}^{k} \rho^{k} \Gamma(2 m+2 k+1)}{\left(\Omega_{i}+\Omega_{j}\right)^{2 k} k ! \Gamma(m+k+1)}\right. \\
\left.\quad \times F\left(1,2 m+2 k+1 ; m+k+1 ; \frac{\Omega_{i}}{\Omega_{i}+\Omega_{j}}\right)\right\}
\end{aligned}
$$

Furthermore, from $[10,(9.14)]$, we know that

$$
F(a, b ; c ; d) \triangleq \sum_{n=0}^{\infty} \frac{(a)_{n}(b)_{n}}{(c)_{n}} \frac{d^{n}}{n !}
$$

where $(\cdot)_{n}$ is the Pochhammer symbol which is defined by $(y)_{n} \triangleq y(y+1)(y+2) \cdots(y+n-1)$. Substitute $(11),(12),(19),(18)$ into $(15),(10)$ is derived. 